Relations industrielles

Industrial Relations

\title{
White-Collar Workers, ouvrage écrit en collaboration, sous la direction d'Albert A. Blum, New York, Random House, 1971, 225 \\ pp.
}

\section{André Roy}

Volume 27, numéro 3, 1972

URI : https://id.erudit.org/iderudit/028316ar

DOI : https://doi.org/10.7202/028316ar

Aller au sommaire du numéro

Éditeur(s)

Département des relations industrielles de l'Université Laval

ISSN

0034-379X (imprimé)

1703-8138 (numérique)

Découvrir la revue

Citer ce compte rendu

Roy, A. (1972). Compte rendu de [White-Collar Workers, ouvrage écrit en collaboration, sous la direction d'Albert A. Blum, New York, Random House, 1971, 225 pp.] Relations industrielles / Industrial Relations, 27(3), 540-545. https://doi.org/10.7202/028316ar

Tous droits réservés (C Département des relations industrielles de l'Université Laval, 1972
Ce document est protégé par la loi sur le droit d'auteur. L'utilisation des services d’Érudit (y compris la reproduction) est assujettie à sa politique d'utilisation que vous pouvez consulter en ligne.

https://apropos.erudit.org/fr/usagers/politique-dutilisation/ 
White-Collar Workers, ouvrage écrit en collaboration sous la direction d'Albert A. Blum, New York, Random House, 1971, 225 pp.

Il ne s'agit pas ici d'une étude sociologique au sens strict, mais plutôt d'une série de monographies sur le développement et le degré de pénétration du syndicalisme dans divers groupes d'employés de bureau ou, plus exactement, de cols blancs.

Cet ouvrage, qui a été publié sous la direction d'Albert A. Blum, de l'université du Michigan à East Lansing, s'ouvre par un chapitre consacré aux employés de bureau en général dont il est lui-même l'auteur.

$\mathrm{Au}$ départ: deux constatations brutales. Les statistiques rangent 34 millions de travailleurs américains dans la catégorie des cols blancs dont il faut en retrancher 7 pour cent qu'on peut considérer comme des cadres supérieurs, ce qui en laisse une masse énorme de près de 27 millions susceptibles d'adhérer à un syndicat quelconque. Ce sont là des données remontant à l'année 1967, mais l'on estime que les cols bleus encore plus nombreux que les cols blancs leur céderont définitivement la place vers 1975 . Par ailleurs, toujours en 1967, les cols blancs représentaient 16.1 pour cent des effectifs des syndicats et à peine 11 pour cent de tous les salariés de cette catégorie.

Pour l'auteur, le problème se pose crûment : pour continuer à se développer ou, ce qui est plus vrai, pour cesser de perdre du terrain, il faut que les syndicats réussissent à pénétrer davantage dans le milieu des employés de bureau. Mais, quand on considère le passé récent, on se rend compte que la chose n'est pas facile.

Se fondant sur le résultat des votes de représentation syndicale tenus parmi des groupes d'employés de bureau au cours de la période 1957-1968, l'auteur signale que les syndicats en ont perdu plus de la moitié.

A partir de ces constatations générales, Blum analyse ensuite objectivement les facteurs qui peuvent inciter les cols blancs à désirer ou à rejeter le syndicalisme. Les uns sont positifs; d'autres, négatifs. Du côté positif, l'auteur en relève deux qui sont d'ordre économique : l'amélioration des salaires et la sécurité de l'emploi. Depuis la guerre, en effet, les salaires des cols blancs se sont gra- duellement dépréciés par rapport à ceux des cols bleus. D'autre part, l'automation, en outre de rendre les emplois plus impersonnels, moins intéressants, fait peser la menace du chômage sur beaucoup d'employés de bureau. Politiquement, la pénétration syndicale est entravée par l'insuffisance de la législation du travail, principalement en ce qui a trait aux pratiques interdites. Par ailleurs, les employeurs s'efforcent de donner aux cols blancs une orientation antisyndicale et à les convaincre de la valeur de l'économie libérale. Psychologiquement, les employés de bureau ne sont pas tellement favorables au syndicalisme, surtout parce qu'ils voient dans l'adhésion à un syndicat un abaissement possible de leur statut social. Mais il y a un autre facteur important dont il faut tenir compte : l'attitude des syndicats eux-mêmes devant la tâche redoutable de syndicaliser des millions de cols blancs. Il apparaît bien que les idées sont divisées à ce sujet. Certains chefs soutiennent que le syndicalisme pour les employés de bureau est une question de pain et de beurre comme pour le reste des salariés; d'autres soutiennent - et c'était, entre autres, l'opinion de Walter Reuther - que, si l'on veut vraiment faire participer les cols blancs à l'action syndicale, il faut faire appel à un certain idéal social qui dépasse le mieux-être matériel de l'individu, leur donner une motivation plus générale, plus haute. En outre, il faut souligner certaines difficultés d'ordre pratique qui sont nées de la rivalité des différents syndicats et qui demeurent toujours aigues. La plupart des grands syndicats industriels cherchent à recruter les cols blancs travaillant dans les limites de leur compétence. Par ailleurs, les syndicats spécialisés ont énormément de difficultés à se tailler une place. C'est le cas notamment du syndicat international des employés de bureau.

Voilà pour la toile de fond. Sur celle-ci viennent se greffer des monographies intéressantes, soit parce que le syndicat sous étude joue un rôle ici mềme au Canada, soit parce que les problèmes rencontrés dans le secteur d'activité en cause sont plus ou moins identiques.

Dans une première monographie, Martin Estey, de l'université de Pennsylvanie, étudie le cas des commis. Plusieurs syndicats sont actifs dans ce secteur. Le plus important est sans contredit celui des commis de détail qui groupe plus de la moitié des membres. Mais il y a 
aussi le syndicat des bouchers et celui des camionneurs dont il faut tenir compte. C'est au sein de cette catégorie de salariés que le syndicalisme a fait le plus de progrès depuis une vingtaine d'années. Mais la place est bien loin d'être convenablement occupée. Les syndicats groupent dans ce secteur de l'activité économique un peu moins d'un million de membres, mais il $y$ a une masse de salariés évaluée à plus de huit millions dont on peut retrancher environ 15 pour cent qui appartiennent aux cadres. Au surplus, est-il bon d'ajouter que le secteur se développe plus vite que le syndicalisme y progresse.

Le recrutement syndical s'avère difficile parce qu'il s'agit la plupart du temps de petites ou moyennes entreprises n'employant qu'un nombre limité de personnes, surtout des femmes travaillant souvent à temps partiel pour gagner un salaire d'appoint ou de gens qui aspirent à devenir employeurs ou artisans.

Il reste néanmoins que le syndicalisme s'est développé dans ce domaine plus qu'ailleurs où il a régressé. L'auteur l'explique principalement par la multiplication des supermarchés alimentaires et des magasins libre-service dans les centres d'achats colossaux qui se sont fixés à la périphérie des grandes villes.

Mais il se pose à ce sujet une question d'un autre ordre. En effet, on peut se demander quelle y est la proportion des cols blancs véritables. Il semble bien que ce soit une minorité, car le personnel de ces établissements est surtout composé d'hommes de métier, des bouchers, de manutentionnaires préposés à la réception, à l'installation et à la livraison de la marchandise, donc des cols blancs sales (dirty white-collars) pour emprunter l'expression de l'auteur. Si le syndicalisme, un syndicalisme tout de même conservateur, assez près des employeurs, peut y croître, c'est qu'il s'agit le plus souvent d'une main-d'œuvre masculine homogène sans beaucoup de formation professionnelle employée dans un type d'entreprises où les chances d'avancement sont réduites au minimum et où il n'existe généralement aucun stimulant.

La syndicalisation des employés du commerce gagnera-t-elle d'autres secteurs? Les syndicats bataillent pour pénétrer dans les magasins à rayons multiples qui emploient des milliers de personnes ainsi que dans les magasins à succursales multiples. S'il y a succès de ce côté, il sera possible d'affirmer qu'un pas important a été fait dans la syndicalisation des cols blancs, mais l'auteur ne croît pas que la pénétration du syndicalisme dans les supermarchés alimentaires puisse être considérée comme une percée dans le monde des cols blancs.

La deuxième monographie traite de la situation du syndicalisme dans les milieux professionnels. L'auteur, James W. Kuhn, de l'université de Columbia, présente le cas des ingénieurs. Aux Etats-Unis, chez ce groupe, le syndicalisme a connu une période de croissance, il y a une vingtaine d'années, qui fut suivie par la suite d'un déclin marqué. Les syndicats formés par les ingénieurs sont indépendants. On en trouve qu'un petit nombre qui sont affiliés aux centrales. Comment expliquer cette montée, puis ce déclin ?

A la fin de la guerre, employeurs et ingénieurs étaient inquiets. Ils redoutaient que les Conseils des relations du travail incluent les groupes d'ingénieurs dans les unités de négociation générales. Ceci, évidemment, ne plaisait ni aux uns ni aux autres. C'est pourquoi, en l'espace de quelques années, un bon nombre d'ingénieurs se groupèrent en syndicats. Mais une fois ce danger disparu, le recrutement cessa presque. Aujourd'hui, s'il reste encore pas mal d'ingénieurs qui sont membres des syndicats, c'est que les vieux adhérents n'ont pas lâché, mais les jeunes ingénieurs n'y voient aucun intérêt. Ils s'y opposent même au nom du professionnalisme. Il n'est pas séant, disent-ils, qu'un ingénieur soit assujetti à une convention collective de travail.

Et pourtant, pourquoi les ingénieurs plus âgés conservent-ils leur adhésion? L'enquête de l'auteur lui a permis d'en déceler certaines raisons : c'est que, tout de même, l'action collective des syndicats a permis aux ingénieurs d'améliorer leurs traitements et d'obtenir certains avantages sociaux; c'est aussi que, à cause des progrès rapides des techniques, la valeur professionnelle des ingénieurs a tendance à décroître rapidement; c'est que, principalement dans les très grandes entreprises, le travail de génie n'est pas tellement créatif et devient souvent simple affaire de vérification; c'est que, enfin, les ingénieurs les plus âgés redoutent advenant une récession, être mis à pied ou qu'ils voient, la quarantaine arrivée, leurs chances d'accéder à des tâches de direction s'estomper rapidement. 
Fondamentalement, il semble, par ailleurs, que ce qui explique le recul du syndicalisme, c'est l'impossibilité dans laquelle se trouvent les syndicats de négocier de véritables conventions collectives par la faute même des intéressés qui comptent sur leur compétence personnelle pour améliorer leurs conditions de vie, d'où il découle que le principal avantage du syndicat, selon les propos d'un ingénieur «c'est de mettre nos problèmes sous le nez de la Compagnie, afin qu'elle ne nous oublie pas $\gg$. Cependant, dans certains cas, le syndicat joue un rôle plus utile. L'auteur cite le cas d'une entreprise assez mal administrée, où le syndicat, par son action, a réussi à établir un meilleur climat, non seulement en réglant les griefs de ses membres, mais en participant à plusieurs autres activités en matière d'évaluation des emplois, d'études scientifiques des coûts, etc. .

L'étude du cas des ingénieurs indique que le développement du syndicalisme dans la plupart des catégories professionnelles peut piétiner longtemps à moins que des conditions économiques défavorables n'obligent les employeurs à resserrer les cordons de la bourse.

Parmi les groupes de travailleurs intellectuels qui, les premiers, ont songé à recourir au droit d'association aux Etats-Unis, il faut signaler le cas des enseignants. Westley A. Wildman, de l'université de Chicago, y consacre une étude fort captivante qui revêt un intérêt d'autant plus grand que, au Québec même, le syndicalisme des enseignants défraie beaucoup l'actualité depuis quelques années.

L'histoire de l'action collective de cette catégorie de travailleurs a commencé tôt. Dès le milieu du XIXe siècle, il existait déjà un certain nombre d'associations d'enseignants, assez en tout cas pour que, en 1857, se fonde la National Education Association. Il ne faut pas s'imaginer qu'il s'agissait là de véritables syndicats non plus que d'une véritable centrale syndicale. L'association avait pour fin essentielle, au départ, de susciter de l'intérêt dans la population pour les choses de l'éducation mais, en même temps, elle ne pouvait que tendre à donner une image plus sympathique de l'instituteur, à valoriser son statut social, à poser les cadres d'une véritable profession. Il fallut beaucoup de temps, en effet, avant que les associations se transforment en syndicats conventionnels et recourent aux mécanismes des contrats collectifs de travail. En 1902, une association concurrente se fonda qui, elle, avait une allure plus nettement syndicale : l'American Federation of Teachers qui s'affilia bientôt à la Fédération américaine du travail. Toutefois, elle eut bien de la difficulté à se faire accepter. En réalité, à la veille de la guerre, l'American Federation of Teachers ne comptait pas 30,000 membres tandis que les effectifs de la National Education Association étaient de 200,000 membres. Il n'existait guère de conventions collectives encore, mais, l'idée avait fait son chemin. Dès 1918, un ancien président de la NEA déclarait: "Si les contribuables ne veulent pas prendre leurs responsabilités, il n'y a qu'une chose à faire : que les enseignants, par leur action concertée et le recours à la négociation collective, forcent les gens à se réveiller $\gg$.

Après la guerre, la négociation de conventions collectives fit son apparition. A Pawtucket, dans le Rhode Island, une section locale de l'AFT força le conseil scolaire à négocier. Il en fut cle même un peu plus tard à Chicago. Le mouvement était lancé. Il prit bientôt de l'ampleur dans les milieux urbains. L'AFT progressait ainsi rapidement; elle ne pouvait que se heurter à NEA qui avait temporisé, mais dût se rendre à l'évidence que, si elle n'entrait pas dans le jeu, elle verrait bientôt ses effectifs décroître. Cette rivalité stimula l'organisation au sein des groupes d'enseignants. Les statistiques prennent ici tout leur sens, puisqu'en 1967-1968, à peu près 300,000 enseignants américains étaient assujettis à 600 conventions collectives conclues en bonne et due forme. L'auteur énumère toute une série de motifs pour expliquer ce phénomène : les enseignants veulent des traitements plus élevés; l'augmentation des enseignants masculins a réduit le virement de personnel; les enseignants veulent participer à l'élaboration des programmes; une lé:gislation élargie leur a octroyé le droit de négociation; la rivalité syndicale a stimulé le militantisme; l'institution de conseils scolaires gigantesques a fait naître des problèmes épineux pour les enseignants; leur activité syndicale accrue s'inscrit dans un contexte culturel nouveau.

Ceci a évidemment entraîné certains ajustements. Au début du syndicalisme, les associations d'enseignants groupaient aussi bien les cadres de l'enseignement que le personnel ordinaire, mais rapide- 
ment, l'American Federation of Teachers a adopté la politique typiquement syndicale de rejeter de ses rangs les surveillants et les administrateurs, tandis que la National Education Association se montre toujours plus accueillante.

Mais c'est dans le domaine de la convention collective principalement qu'il est intéressant de considérer les observations de l'auteur. Celles-ci touchent trois sujets principaux : les questions pécuniaires, les conditions de travail, les affaires professionnelles.

Les syndicats ont évidemment mis l'accent sur les questions salariales. Au départ, la grève aidant, il y a eu des améliorations sensibles, mais, peu à peu, les employés des services publics en général eurent également vite fait de recourir aux mêmes moyens, de sorte qu'il faut prévoir une certaine stabilisation dans l'avenir. Les enseignants, comme les employés des services publics en général, devront faire face à la résistance des contribuables. Par ailleurs, note l'auteur, \& assumant que les enseignants et les autres groupes d'employés des administrations publiques continuent à faire la grève, et à la faire avec succès, l'Etat peut trouver souhaitable et nécessaire de centraliser à son niveau les décisions en matière de salaire et sur d'autres questions fondamentales. $\mathrm{Si}$, par exemple, les enseignants des gros conseils scolaires à l'intérieur d'un Etat sont en mesure de faire avec succès la grève au détriment des petits et si les gros et puissants syndicats de fonctionnaires travaillant dans des services relativement essentiels, sont en mesure par la grève d'affamer ceux qui occupent des fonctions administratives moins vitales et moins apparentes, les législatures d'Etat peuvent assumer directement le rôle d'agent de négociation du côté de l'employeur ». Le but d'une pareille orientation serait double : en ce qui touche l'éducation, établir la parité parmi tous les conseils scolaires, quelle qu'en soit l'importance, à l'intérieur d'un Etat donné pour ce qui touche l'ensemble des employés des services gouvernementaux à l'intérieur d'un Etat, établir la parité des salaires également entre les enseignants et les autres groupes d'employés.

Donc, ce à quoi l'on s'attend aux Etats-Unis ne serait en quelque sorte que la répétition de ce qui se passe au Québec, un peu comme on applique un même remède à un même mal.
En ce qui a trait aux conditions de travail, les syndicats d'enseignants tendent de plus en plus à faire modifier certaines règlementations traditionnelles dans le domaine de l'éducation. Ainsi, la longueur de l'année scolaire, souvent fixée par voie législative, est devenue sujet de négociation de même que les horaires scolaires. Il en est ainsi du rapport maître-élèves. En certains endroits, les enseignants ont obtenu le lunch gratuitement à la cafétaria de l'école en échange de la surveillance des étudiants durant cette période. Mais les enseignants dans l'ensemble tendent surtout à la * professionnalisation » de leur métier et à la réduction des périodes d'enseignement pour leur permettre d'accorder plus de temps à la préparation de leurs cours. Dans le domaine pédagogique lui-même, les syndicats ont pu obtenir des concessions importantes, par exemple, le droit de contester les décisions de la direction sur les questions pédagogiques, le droit d'élire les principaux au niveau des écoles, le droit de participer à l'évaluation du degré de compétence de l'enseignant, . etc.

A la fin de son étude, l'auteur tente de faire une analyse de ce qu'il appelle la dynamique des négociations dans le milieu scolaire et de prévoir les transformations susceptibles de s'y produire par suite de l'extension du syndicalisme en milieu scolaire.

Sur le premier point, il estime que pour l'instant les parties jouent le rôle des agents de négociation ordinaire qui s'engagent dans la voie des compromis, que le syndicat d'enseignants se considère comme une institution politique qui a quelque chose à apporter à ses adhérents, à cause de la rivalité entre les deux associations existantes. Sur le deuxième point, il faut s'attendre que, peu à peú, les enseignants devront sacrifier les échelles de salaires uniformes et qu'on assistera à une différenciation de plus en plus marquée où sera davantage tenu compte de la compétence et de l'efficacité. Il se peut aussi qu'il y ait une tendance de plus en plus marquée vers l'automatisation de l'enseignement par l'utilisation de la télévision scolaire et de l'informatique, même si les projets en ce sens ont marqué le pas depuis quelques années.

La dernière monographie, qui a pour auteur Léo Troy traite du rôle joué par le syndicalisme dans le fonctionnarisme fédéral. Il s'agit d'un phénomène ré. 
cent. A venir jusqu'au fameux décret 10988 du président Kennedy, le syndicalisme n'existait à vrai dire que parmi les employés des postes et ceux d'un certain nombre d'agences gouvernementales.

On ne saurait sous-estimer l'importance de ce décret qui a pour objet de protéger le droit d'association des employés du gouvernement fédéral américain. Cependant, certaines dispositions posent des problèmes très ardus. Il $\mathrm{y} a$ d'abord la disposition relative au conflit d'intérêt qui, tout en permettant à certaines catégories d'employés d'être membres des associations, leur interdit le droit d'éligibilité aux postes de direction ainsi que celui d'agir comme représentants syndicaux. Cette stipulation qui a reçu une interprétation très large, a pour effet de priver les syndicats de ses éléments les meilleurs et les plus dynamiques. Autre question majeure : celle de l'établissement des unités de négociations. Il y a interdiction de former des groupes mixtes, c'est-à-dire comprenant le personnel d'exécution et le personnel qui occupe des fonctions de nature administrative ainsi que le droit pour les professionnels d'avoir leurs propres groupes distincts. Par ailleurs, on trouve différents types de reconnaissance, la ro connaissance informelle qui permet à l'association de représenter ses membres, mais non d'être un véritable agent de négociation, la reconnaissance formelle que peuvent obtenir les groupes minoritaires ou ceux qui ne désirent pas de conventions collectives écrites, enfin, la représentation exclusive et la négociation de véritables conventions mais limitées à certaines questions d'abord et dépourvues de mécanismes valables pour trancher les différends parce qu'il n'existe pas d'arbitrage à sentence exécutoire et que la grève est prohibée.

Malgré ces entraves, les effectifs représentés par les syndicats ont plus que doublé entre 1962 et 1968 , passant de 521,000 à $1,239,000$. Cependant, ceci ne correspond pas au nombre des membres à cause de l'inexistence des clauses de sécurité syndicale qui sont prohibées par le décret gouvernemental. Il n'en reste pas moins qu'il $\mathrm{y}$ a eu un progrès marqué. De décembre 1962 à novembre 1967 , le nombre des travailleurs représentés, à l'exception des employés des postes qui étaient déjà syndiqués de longue date, est passé de 42,000 à 630,000 , soit, pour les cols bleus, de
18,000 à 338,000 et, pour les cols blancs, de 24,000 à 291,000 . Un autre fait notable : le degré de syndicalisation des cols blancs dans les services de l'Etat est beaucoup plus haut que dans l'économie américaine.

Les employés du gouvernement fédéral sont représentés par plusieurs syndicats, mais c'est l'American Federation of Government Employees (AFGE) affilié à la centrale FAT-COI qui est de beaucoup la plus importante, puisqu'elle représente 186,000 des 291,000 cols blancs.

Au premier abord, le tableau apparaît assez reluisant, mais il y a une ombre au tableau. Aux Etats-Unis comme ailleurs, les services gouvernementaux comptent de plus en plus de professionnels qui sont moins enclins à recourir au syndicalisme que les autres catégories, ce qui est de nature à ralentir la syndicalisation, sinon à provoquer une régression. $\mathrm{Au}$ fond, se répéterait au niveau gouvernemental un phénomène semblable à celui qui a été observé chez les ingénjeurs en général. Par ailleurs, il semble que le gouvernement fédéral, suivant en cela une tendance qui ne cesse de s'affirmer d'une façon générale, confiera un nombre de plus en plus grand de tâches à l'entreprise privée sous forme de contrats, ce qui freinera le développement du fonctionnarisme.

L'auteur note aussi trois autres phénomènes prévisibles: le développement d'un antisyndicalisme virulent dans certains secteurs, comme les services armés, par exemple, qui sont farouchement antisyndicaux ; l'augmentation des noirs au sein des services peut déclencher peutêtre une ère de discrimination raciale qui aura des répercussions sur le syndicalisme et enfin, la possibilité que les syndicats de fonctionnaires engagent la bataille dans le but de faire reconnaître leurs droits de participation à l'action politique.

Quelles conclusions générales tirer de ces études? Que le syndicalisme, depuis ses origines, n'a progressé que lentement parmi les cols blancs, d'une façon générale, même s'il a enregistré des gains appréciables au cours des vingt dernières années, notamment dans le secteur des services publics. Cependant, il faut noter qu'il n'est que marginal dans l'entreprise privée. Quand l'on sait que, d'une part, le secteur tertiaire, immense réservoir de cols blancs, se développe sans cesse aux dépens du secteur secondaire et que 
celui-là comptera peut-être soixante-dix pour cent de toute la main-d'œuvre d'ici une dizaine d'années, il ne faut pas se surprendre, d'autre part, que le pourcentage de travailleurs syndiqués par rapport à la main-d'œuvre totale ait décliné de quelque sept pour cent au cours des dernières années et que le mouvement syndical devra, s'il veut remonter la pente, relever un défi d'envergure.

\section{André ROY}

D. O. Sewell, Training the Poor: $\mathbf{A}$ Benefit-Cost Analysis of Manpower Programmes in the U.S. Anti-Poverty Programme (Kingston: Queen's University, Industrial Relations Centre 1971). XIV + 153

It is a shame that the title of this book may keep it from being read by a wider audience. Upon receiving it I thought it would be yet another of those dreary, specialized little empirical studies that are quickly read and deservedly forgotten.

The Sewell book, however, is not one of these. In fact a more descriptive, but clumsy, title would have been, «The Theory and Practice of Cost-Benefit Analysis - with Special Reference to the U.S. Anti-Poverty Programme ». Fully one-half of the text is devoted to the conceptual problems of cost-benefit analysis dealing with such questions as allocative versus distributive effects of training, the role of externalities, and separation of the real, from the pecuniary effects of training.

In dealing with these and other conceptual problems, Sewell has been exhaustive in surveying the literature right up to the date of publication. There is an excellent seven page selective bibliography, and the quality of discussion in the text is ample proof that Sewell has in fact read the material cited in his bibliography.

While the first half of the book will be of interest to a very wide audience including academics and administrators with some background in economics the second half will be primarily of interest to those formulating and administering training programmes in the field.

Sewell has set out to determine the benefits and costs of a very special training project sponsored under the U.S.
Manpower Development and Training Act (M.D.T.A.) of 1962. The special project known as Manpower Improvement Through Community Effort (M.I.T.C.E.) was conducted in North Carolina, and Sewell examined the subsequent earnings profiles of workers training during the contract year 1965-66.

In accepting the conclusions one should be careful not to interpret them as the values that might be obtained for training under Canada's Manpower Retraining Act. Eligibility requirements for M.I.T.C.E. training are stringent : « Trainess were required to be heads of households, at least 18 years of age, and prior to enrolment in training they had to have been unemployed for more than six months, or to have been members of farm families with incomes of less than $\$ 1,200$ per annum. $\gg$ Certainly we are not training the cream of the crop here.

But not only were the trainess suffering from poverty, they were also nonwhite in predominant numbers $(88 \%)$. Sewell is clearly studying the training of a severely disadvantaged group. Perhaps, then, his results would be comparable in Canada with the results from a training project involving Indians.

Sewell conducted a questionnaire survey among three hundred and sixty of the total five hundred and thirty-four male and female trainees in 1965-66, sixteen months after they had completed training. Roughly two-thirds of the trainees received on-the-job training, and the other third received institutional training. Basically, what was done was to compare the trainees pre, and posttraining earnings with the earnings of a sample of non-trainees possessing otherwise similar characteristics. Some of Sewell's conclusions are interesting; but space allows for a listing of some only.

Even though the trainees represented one of the most disadvantaged segments of the population in the U.S., Sewell concludes that their training added more to national output, through increased productivity, than to cost. The aggregate allocative benefit-cost ratio is calculated at 3.1 ; that is, for every dollar sunk into training, three dollars is generated because of increased worker productivity. Moreover, Sewell believes that the income gains were substantial enough to lift most of the trainees out of poverty. 